\title{
Irregular Sampling Theorems for Wavelet Subspaces
}

\author{
Wen Chen, Shuichi Itoh, Member, IEEE, and Junji Shiki
}

\begin{abstract}
From the Paley-Wiener 1/4-theorem, the finite energy signal $f(t)$ can be reconstructed from its irregularly sampled values $f\left(k+\delta_{k}\right)$ if $f(t)$ is band-limited and $\sup _{k}\left|\delta_{k}\right|<1 / 4$. We consider the signals in wavelet subspaces and wish to recover the signals from its irregular samples by using scaling functions. Then the way to estimate the upper bound of $\sup _{k}\left|\delta_{k}\right|$ such that the irregularly sampled signals can be recovered is very important. Following the work done by Liu and Walter, we present an algorithm which can estimate a proper upper bound of $\sup _{k}\left|\delta_{k}\right|$. Compared to Paley-Wiener 1/4-theorem, this theorem can relax the upper bound for sampling in some wavelet subspaces.
\end{abstract}

Index Terms-Biorthogonality, MRA, orthogonality, sampling, scaling function, wavelet, Zak-transform.

\section{INTRODUCTION}

$\mathbf{F}$ OR a finite-energy $\gamma$-band continuous signal $f(t), t \in$ $R$, i.e., $f \in L^{2}(R)$ and $\operatorname{supp} \hat{f}(\omega)=[-\gamma, \gamma]$, the classical Shannon Sampling Theorem gives the following reconstruction formula:

$$
f(t)=\sum_{n} f(n T) \frac{\sin \gamma(t-n T)}{\gamma(t-n T)}
$$

where $T \leq \pi / \gamma$ and $\hat{f}(\omega)$ is the Fourier transform of $f(t)$ defined by

$$
\hat{f}(\omega)=\int_{R} f(t) e^{-i \omega t} d t
$$

Unfortunately it is not appropriate for nonbandlimited signals. However, if we let $\gamma=2^{m} \pi, m \in Z$, the problem can be viewed as that of sampling in a wavelet subspace, with $\varphi(t)=\sin \pi t / \pi t$ playing the role of scaling function of Multi-Resolution Analysis (MRA) $\left\{V_{m}=\overline{\operatorname{span}}\left\{\varphi\left(2^{m} t-n\right)\right\}_{n}\right\}_{m}$. Realizing these properties, Walter [16] extended (1) such that it holds for a class of scaling functions. Let $\varphi(t)$ be a continuous orthonormal scaling function of MRA $\left\{V_{m}\right\}_{m}$ such that $|\varphi(t)| \leq O\left(|t|^{1+\varepsilon}\right)$ for some $\varepsilon>0$. Walter [16] showed that there is a sequence $\left\{S_{k}(t)\right\}_{k}$ in $V_{0}$ such that $S_{k}(t)=S_{0}(t-k)$ and

$$
f(t)=\sum_{k} f(k) S_{0}(t-k)
$$

holds for any $f(t) \in V_{0}$.

However, in many cases the sampling is not always at the same step. How should irregular sampling cases be dealt with?

Manuscript received July 11, 1996; revised December 15, 1997. The material in this paper was presented in part at the IEEE International Symposium on Information Theory, Ulm, Germany, June 29-July 4, 1997.

The authors are with the Department of Information Network Sciences, Graduate School of Information Systems, University of ElectroCommunications, Chofugaoka 1-5-1, Chofu, Tokyo 182, Japan.

Publisher Item Identifier S 0018-9448(98)02351-7.
Paley-Wiener's $1 / 4$-Theorem (see [18, p. 151]) states that, if $\sup _{k}\left|\delta_{k}\right|<1 / 4$ and $\delta_{k}=-\delta_{-k}$ then

$$
f(t)=\sum_{k} f\left(k+\delta_{k}\right) \frac{G(t)}{G^{\prime}\left(k+\delta_{k}\right) G\left(t-\left(k+\delta_{k}\right)\right)}
$$

holds for any $f(t) \in P_{\pi}$ (Paley-Wiener Space), where

$$
G(t)=t \prod_{n=1}^{\infty}\left(1-t^{2} /\left(n+\delta_{n}\right)^{2}\right) .
$$

But it cannot deal well with nonbandlimited signals, and sampling with the symmetricity constraint $\delta_{k}=-\delta_{-k}$ is also restrictive. Following Walter's [16] work, Liu and Walter [11] tried to extend Paley-Wiener's 1/4-Theorem to hold for the sampling in a class of orthonormal wavelet subspaces without the symmetric sampling constraint $\delta_{k}=-\delta_{-k}$. But they could not claim that there is a sequence $\delta_{\varphi} \subset(0,1]$ such that a similar to (3) reconstruction formula holds when $\sup _{k}\left|\delta_{k}\right|<$ $\delta_{\varphi}$. Then Liu [10] turned to deal with the special case, spline wavelets, by applying the Feichtinger-Grochenig Iterative Algorithm (see [7]). Chen, Itoh, and Shiki [2] obtained a recovering formula for sampling in general wavelet subspaces, but they were led to an $l^{1}$-bound on $\left\{\delta_{k}\right\}_{k}$. In fact, they cannot yet estimate the aforementioned $\delta_{\varphi}$.

In this paper, we can estimate an $l^{\infty}$-bound $\delta_{\varphi}$ for $\left\{\delta_{k}\right\}_{k}$, which enables a reconstruction formula similar to (3) to hold when $\sup _{k}\left|\delta_{k}\right|<\delta_{\varphi}$. Our theorem does not only require the symmetric sampling constraint $\delta_{k}=-\delta_{-k}$, but also relaxes the bound $\delta_{\varphi}$ for the sampling in some wavelet subspaces. In summary, we can estimate some $\delta_{\varphi} \in(0,1]$, such that for any $\left\{\delta_{k}\right\}_{k}$ with $\sup _{k}\left|\delta_{k}\right|<\delta_{\varphi}$, there is an $\left\{S_{k}(t)\right\}_{k} \subset V_{0}$ such that

$$
f(t)=\sum_{k} f\left(k+\delta_{k}\right) S_{k}(t)
$$

holds for any $f(t) \in V_{0}$. Our idea is to let

$$
q_{\varphi}(s, t)=\sum_{n} \varphi(s-n) \varphi(t-n) .
$$

Then we show that $\left\{q_{\varphi}(k, t)\right\}_{k}$ is a Riesz basis of $V_{0}$. By applying the Paley-Wiener Theorem (see $[18$, p. 38]), we manage to find some $\delta_{\varphi}$ so that $\left\{q_{\varphi}\left(k+\delta_{k}, t\right)\right\}_{k}$ is another Riesz basis of $V_{0}$ equivalent to $\left\{q_{\varphi}(k, t)\right\}_{k}$. Then there is a basis sequence $\left\{S_{k}(t)\right\} \subset V_{0}$ biorthogonal to $\left\{q_{\varphi}\left(k+\delta_{k}, t\right)\right\}_{k}$, such that

$$
f(t)=\sum_{k} S_{k}(t) \int_{R} f(t) q_{\varphi}\left(k+\delta_{k}, t\right) d t
$$

holds for any $f(t) \in V_{0}$. Since

$$
\int_{R} f(t) q_{\varphi}\left(k+\delta_{k}, t\right) d t=f\left(k+\delta_{k}\right)
$$

holds, we obtain (4). 
Furthermore, the theorems are modified to be appropriate for the shift sampling case by using Zak-transform (see [9]). Later, we also calculate some examples and indicate that $\delta_{\varphi}$ can be bigger than 1/4 for the sampling in B-spline of order 1 wavelet subspace.

Let us now introduce MRA (Multi-Resolution Analysis) which was mentioned above. (For more details see [13], [14], or any book on wavelets such as [1], [15], and [17].)

A subspace sequence $\left\{V_{m}\right\}_{m}$ of $L^{2}(R)$ is said to be an MRA if

1) $V_{m} \subset V_{m+1}, \cap_{m} V_{m}=\{0\}, \overline{\cup_{m} V_{m}}=L^{2}(R)$,

2) Function $f(t) \in V_{m}$ if and only if $f(2 t) \in V_{m+1}$,

3) There is a function (called scaling function) $\varphi(t) \in V_{0}$ such that $\{\varphi(t-k)\}_{k}$ form a Riesz basis in $V_{0}$.

The terms Multi-Resolution Approximation and MultiResolution Decomposition are also used sometimes. If $\{\varphi(t-k)\}_{k}$ is an orthogonal (respectively, orthonormal) Riesz basis, MRA $\left\{V_{m}\right\}_{m}$ and scaling function are considered orthogonal (respectively, orthonormal).

MRA pair $\left\{V_{m}, \tilde{V}_{m}\right\}_{m}$ are considered biorthogonal if

$$
\int_{R} \varphi(t-k) \tilde{\varphi}(t-l) d t=\delta_{k l}
$$

where $\varphi(t) \in V_{0}$ and $\tilde{\varphi}(t) \in \tilde{V}_{0}$ are scaling functions of MRA $\left\{V_{m}\right\}_{m}$ and $\left\{\tilde{V}_{m}\right\}_{m}$, respectively. In that case, scaling function pair $\{\varphi(t), \tilde{\varphi}(t)\}$ is also considered biorthogonal.

Finally, let us introduce some notations used in this paper. For a measurable set $E \subset R,|E|$ denotes the measure of $E$. For the measurable functions $f(t)$ and $g(t)(t \in R)$, a real number $\lambda>0$, and an interval $[a, b] \subset[-1,1]$, we write

$$
\begin{aligned}
l^{1} & =\left\{\left\{a_{k}\right\}_{k}\left|\sum_{k}\right| a_{k} \mid<\infty\right\} \\
l^{2} & =\left\{\left.\left\{a_{k}\right\}_{k}\left|\sum_{k}\right| a_{k}\right|^{2}<\infty\right\} \\
\|f\| & =\left(\int_{R}|f(t)|^{2} d t\right)^{1 / 2} \\
\|f\|_{L^{2}[0,2 \pi]} & =\left(\int_{0}^{2 \pi}|f(t)|^{2} d t\right)^{1 / 2} \\
\|f\|_{\infty} & =\inf _{|E|=0} \sup _{R \backslash E}|f(t)| \\
\|f\|_{0} & =\sup _{|E|=0} \inf _{R \backslash E}|f(t)| \\
\hat{f}^{*}(\omega) & =\sum_{n} f(n) e^{i n \omega} \\
G_{f}(\omega) & =\left(\sum_{k}|\hat{f}(\omega+2 k \pi)|^{2}\right)^{1 / 2} \\
q_{f, g}(s, t) & =\sum_{n} f(s-n) g(t-n) \\
L^{2}(R) & =\{f|\|f\||<\infty\} \\
L^{\infty}(R) & =\left\{f \mid\|f\|_{\infty}<\infty\right\} \\
q_{f}(s, t) & =\sum_{n} f(s-n) f(t-n)
\end{aligned}
$$

$$
\begin{aligned}
q_{f, g}(s, t)= & \sum_{n} f(s-n) g(t-n) \\
\operatorname{Lip}^{\lambda}= & \left\{f|| f(s)-f(t)\left|\leq C_{f}\right| s-\left.t\right|^{\lambda}, s, t \in R\right\} \\
\operatorname{Lip}_{[a, b]}^{\lambda}(s)= & \left\{f|| f(s+h)-\left.f(s)\left|\leq C_{f}(s)\right| h\right|^{\lambda},\right. \\
& h \in[a, b]\}, \\
\|f\|_{\operatorname{Lip}_{[a, b]}^{\lambda}(\mathrm{s})}= & \sup _{[a, b]} \frac{|f(s+h)-f(s)|}{|h|^{\lambda}},
\end{aligned}
$$$$
\text { for } f(t) \in \operatorname{Lip}_{[a, b]}^{\lambda}(s) \text {. }
$$

\section{IRREgUlar SAMPLING THEOREM AND ALGORITHM FOR ORTHOGONAL WAVELET SUBSPACES}

Firstly, we shall show the existence of the $\delta_{\varphi}$ described in Section I for the sampling in orthogonal wavelet subspaces. Then we shall provide an algorithm to estimate the $\delta_{\varphi}$.

Theorem 1: Let $\varphi(t)$ be a continuous orthogonal scaling function of MRA $\left\{V_{m}\right\}_{m}$ with

1) $|\varphi(t)|=O\left(1 /|t|^{1+\varepsilon}\right)$ for some $\varepsilon>0$,

2) $\hat{\varphi}^{*}(\omega) \neq 0$.

Then there exists a $\delta_{\varphi} \in(0,1]$, such that for any $\left\{\delta_{k}\right\}_{k} \subset$ $\left[-\delta_{\varphi}, \delta_{\varphi}\right]$ there is a sequence $\left\{S_{k}(s)\right\}_{k}$ biorthogonal to $\left\{q_{\varphi /\|\varphi\| \|}\left(s, k+\delta_{k}\right)\right\}_{k}$ in $V_{0}$ such that (4) holds for any $f(t) \in V_{0}$.

In order to demonstrate the theorem, we need a lemma which can be found in Liu and Walter [11].

Lemma 1: Let $\varphi(t)$ be an orthonormal continuous scaling function of MRA $\left\{V_{m}\right\}_{m}$ with $|\varphi(t)|=O\left(1 /|t|^{1+\varepsilon}\right)$ for some $\varepsilon>0$. Then

$$
f(t)=\int_{R} f(s) q_{\varphi}(s, t) d s
$$

holds for any $f \in V_{0}$.

Proof of Theorem: Let $g(t)=\varphi(t) /\|\varphi\|$. Then, $g(t)$ is an orthonormal continuous scaling function with $|g(t)|=$ $O\left(1 /|t|^{1+\varepsilon}\right)$ for some $\varepsilon>0$ and $\hat{g}^{*}(\omega) \neq 0$.

Define

$$
q_{g}(s, t)=\sum_{n} g(s-n) g(t-n) .
$$

Then, Walter [16] tells us that $\left\{q_{g}(s, k)\right\}_{k}$ is a Riesz basis in $V_{0}$, i.e., for any $\left\{c_{k}\right\}_{k} \in l^{2}$,

$$
C^{-1} \sum_{k}\left|c_{k}\right|^{2} \leq\left\|\sum_{k} c_{k} q_{g}(s, k)\right\|^{2} \leq C \sum_{k}\left|c_{k}\right|^{2}
$$

holds for some $C \geq 1$. If we can find a $\delta_{\varphi} \in(0,1]$, such that for any $\left\{\delta_{k}\right\}_{k} \in\left[-\delta_{\varphi}, \delta_{\varphi}\right]$, there is a $\theta \in[0,1)$ such that

$$
\begin{aligned}
& \| \sum_{k} c_{k} q_{g}\left(s, k+\delta_{k}\right)- \sum_{k} c_{k} q_{g}(s, k) \|^{2} \\
& \leq \theta\left\|\sum_{k} c_{k} q_{g}(s, k)\right\|^{2}
\end{aligned}
$$

holds for any $\left\{c_{k}\right\}_{k} \in l^{2}$, then $\left\{q_{g}\left(s, k+\delta_{k}\right)\right\}_{k}$ is a Riesz basis in $V_{0}$ due to Paley-Wiener Theorem (see [18, p. 38]). 
Hence, there is a sequence $\left\{S_{k}(s)\right\}_{k}$ in $V_{0}$ biorthogonal to $\left\{q_{g}\left(s, k+\delta_{k}\right)\right\}_{k}$, such that

$$
f(t)=\sum_{k} S_{k}(t) \int_{R} f(s) q_{\varphi}\left(k+\delta_{k}, s\right) d s
$$

holds for any $f(t) \in V_{0}$, i.e., (4) holds for any $f(t) \in V_{0}$ due to Lemma 1.

In order to show (9), let

$$
\begin{aligned}
\Delta= & \left\|\sum_{k} c_{k} q_{g}\left(s, k+\delta_{k}\right)-\sum_{k} c_{k} q_{g}(s, k)\right\|^{2} \\
= & \left\|\sum_{n}\left(\sum_{k} c_{k}\left(g\left(k+\delta_{k}-n\right)-g(k-n)\right)\right) g(s-n)\right\|^{2} \\
= & \sum_{n}\left|\sum_{k} c_{k}\left(g\left(k+\delta_{k}-n\right)-g(k-n)\right)\right|^{2} \\
= & \sum_{n} \sum_{k, l}\left(g\left(k+\delta_{k}-n\right)-g(k-n)\right) \\
& \cdot\left(g\left(l+\delta_{l}-n\right)-g(l-n)\right) c_{k} c_{l} \\
= & \sum_{k, l}\left(\sum_{n}\left(g\left(k+\delta_{k}-n\right)-g(k-n)\right)\right. \\
& \left.\quad\left(g\left(l+\delta_{l}-n\right)-g(l-n)\right)\right) c_{k} c_{l}
\end{aligned}
$$

where (11) is due to the orthonormality of $g(s)$. Denote

$a_{k l}=\sum_{n}\left(g\left(k+\delta_{k}-n\right)-g(k-n)\right)\left(g\left(l+\delta_{l}-n\right)-g(l-n)\right)$

then $a_{k l l}=a_{l k}$ and

$$
\begin{aligned}
\Delta & =\sum_{k, l} a_{k l} c_{k} c_{l} \\
& \leq \sum_{k, l}\left|a_{k l}\right|\left(c_{k}^{2}+c_{l}^{2}\right) / 2 \\
& =\frac{1}{2}\left(\sum_{k}\left(\sum_{l}\left|a_{k l}\right|\right) c_{k}^{2}+\sum_{l}\left(\sum_{k}\left|a_{k l}\right|\right) c_{l}^{2}\right) \\
& =\sum_{k}\left(\sum_{l}\left|a_{k l}\right|\right) c_{k}^{2} \\
& \leq\left(\sup _{k} \sum_{l}\left|a_{k l}\right|\right) \sum_{k} c_{k}^{2} .
\end{aligned}
$$

But

$$
\begin{aligned}
\sup _{k} & \sum_{l}\left|a_{k l}\right| \\
\leq & \sup _{k} \sum_{l} \sum_{n}\left|g\left(k+\delta_{k}-n\right)-g(k-n)\right| \\
& \cdot\left|g\left(l+\delta_{l}-n\right)-g(l-n)\right| \\
\leq & \sup _{k} \sum_{n}\left|g\left(k+\delta_{k}-n\right)-g(k-n)\right| \\
& \cdot \sum_{l}\left|g\left(l+\delta_{l}-n\right)-g(l-n)\right| \\
\leq & \sup _{k} \sum_{\alpha}\left|g\left(\alpha+\delta_{k}\right)-g(\alpha)\right| \\
& \cdot \sum_{\beta}\left|g\left(\beta+\delta_{\beta+k-\alpha}\right)-g(\beta)\right|
\end{aligned}
$$

where (13) is due to the index transform $\alpha=k-n$ and $\beta=l-n$.

In the meantime, the assumption $|g(t)|=O\left(1 /|t|^{1+\varepsilon}\right)$ for some $\varepsilon>0$ and the continuity of $g(t)$ imply that the series

$$
\sum_{n}|g(n+t)|
$$

converges uniformly with respect to $t$ on $[-1,1]$, and

$$
\sum_{\beta}\left|g\left(\beta+\delta_{\beta+k-\alpha}\right)-g(\beta)\right|
$$

is uniformly bounded with respect to $\left\{\delta_{k}\right\}_{k} \subset[-1,1]$. Hence,

$$
\begin{aligned}
\lim _{\delta_{\varphi} \rightarrow 0} \sum_{\alpha}\left|g\left(\alpha+\delta_{k}\right)-g(\alpha)\right| \\
=\sum_{\alpha} \lim _{\delta_{\varphi} \rightarrow 0}\left|g\left(\alpha+\delta_{k}\right)-g(\alpha)\right|=0
\end{aligned}
$$

and

$$
\begin{aligned}
\sum_{\beta}\left|g\left(\beta+\delta_{\beta+k-\alpha}\right)-g(\beta)\right| \\
\quad \leq \sup _{\alpha, k} \sum_{\beta}\left|g\left(\beta+\delta_{\beta+k-\alpha}\right)-g(\beta)\right|<\infty .
\end{aligned}
$$

The above argument implies that there really exists some $\delta_{\varphi} \in(0,1]$, such that for any $\left\{\delta_{k}\right\}_{k} \subset\left[-\delta_{\varphi}, \delta_{\varphi}\right]$

$$
\sum_{\alpha}\left|g\left(\alpha+\delta_{k}\right)-g(\alpha)\right| \sum_{\beta}\left|g\left(\beta+\delta_{\beta+k-\alpha}\right)-g(\beta)\right| \leq \theta C^{-1} .
$$

That is,

$$
\sup _{k} \sum_{\alpha}\left|g\left(\alpha+\delta_{k}\right)-g(\alpha)\right| \sum_{\beta}\left|g\left(\beta+\delta_{\beta+h-\alpha}\right)-g(\beta)\right| \leq \theta C^{-1} .
$$

From (12), (13), and (17), we derive

$$
\Delta \leq \theta C^{-1} \sum_{k} c_{k}^{2}
$$

Then, due to (8), we conclude that (9) holds.

The theorem tells us that there really exist some $\delta_{\varphi} \in(0,1]$, such that an irregularly sampled signal with deviation $\left\{\delta_{k}\right\}_{k}$. within some interval $\left[-\delta_{\varphi}, \delta_{\varphi}\right]$ can be reconstructed. However, we also need to know how big the $\delta_{\varphi}$ can be, so that we can design a sampling satisfying the criterion for a concrete signal. Therefore, we should find some algorithm to estimate the $\delta_{\varphi}$ from the scaling function $\varphi$. We need at first to introduce a function class $L_{\sigma}^{\lambda}[a, b](\lambda>0, \sigma \in[0,1)$, $0 \in[a, b] \subset[-1,1])$ and to give some simple propositions of that function class, then present the algorithm. 


\section{Definition 1:}

$$
f(t) \in L_{\sigma}^{\lambda}[a, b](\lambda>0, \sigma \in[0,1), 0 \in[a, b] \subset[-1,1])
$$

if there is a constant $C_{\sigma, f}>0$ such that for any $\left\{\delta_{k}\right\}_{k} \subset[a, b]$

$$
\sum_{k}\left|f\left(k+\sigma+\delta_{k}\right)-f(k+\sigma)\right| \leq C_{\sigma, f}\left(\sup _{k}\left|\delta_{k}\right|\right)^{\lambda} \text {. }
$$

We also write

$$
\|f\|_{L_{\sigma}^{\lambda}[a, b]}=\sup _{[a, b]} \frac{\sum_{k}\left|f\left(k+\sigma+\delta_{k}\right)-f(k+\sigma)\right|}{\left(\sup _{k}\left|\delta_{k}\right|\right)^{\lambda}} .
$$

\section{Proposition 1:}

1) $L_{\sigma}^{\lambda}[a, b]=L_{\sigma}^{\lambda}[a, 0] \cap L_{\sigma}^{\lambda}[0, b]$ and $L_{\sigma}^{\lambda}[a, b] \subset L_{\sigma}^{\lambda^{\prime}}[a, b]$ if $\lambda>\lambda^{\prime}$.

2)

$$
\begin{aligned}
\left\{f \mid \sum_{k}\|f\|_{\operatorname{Lip}_{[\mathrm{a}, b]}^{\lambda}(\mathrm{k}+\sigma)}<\infty\right\} & \subset L_{\sigma}^{\lambda}[a, b] \\
& \subset \cap_{k} \operatorname{Lip}_{[a, b]}^{\lambda}(k+\sigma) .
\end{aligned}
$$

3) If $f(t)$ is differentiable on each interval $k+\sigma+[a, b]$ and

$$
\sum_{k} \sup _{k+\sigma+[a, b]}\left|f^{\prime}(t)\right|<\infty
$$

then $f(t) \in L_{\sigma}^{1}[a, b]$.

Proof:

1) Obviously $L_{\sigma}^{\lambda}[a, b] \subset L_{\sigma}^{\lambda}[a, 0]$ and $L_{\sigma}^{\lambda}[a, b] \subset L_{\sigma}^{\lambda}[0, b]$ hold. Therefore, $L_{\sigma}^{\lambda}[a, b] \subset L_{\sigma}^{\lambda}[a, 0] \cap L_{\sigma}^{\lambda}[0, b]$ holds. On the other hand, for any $\left\{\delta_{k}\right\}_{k} \subset[a, b]$

$$
\begin{aligned}
\sum_{k} \mid & f\left(k+\sigma+\delta_{k}\right)-f(k+\sigma) \mid \\
= & \sum_{b \geq \delta_{k} \geq 0}\left|f\left(k+\sigma+\delta_{k}\right)-f(k+\sigma)\right| \\
& +\sum_{a \leq \delta_{k}<0}\left|f\left(k+\sigma+\delta_{k}\right)-f(k+\sigma)\right| \\
\leq & \|f\|_{L_{\sigma}^{\lambda}[0, b]}\left(\sup _{k}\left|\delta_{k}\right|\right)^{\lambda}+\|f\|_{L_{\sigma}^{\lambda}[a, 0]}\left(\sup _{k}\left|\delta_{k}\right|\right)^{\lambda} \\
\leq & \left(\|f\|_{L_{\sigma}^{\lambda}[0, b]}+\|f\|_{L_{\sigma}^{\lambda}[a, 0]}\right)\left(\sup _{k}\left|\delta_{k}\right|\right)^{\lambda} .
\end{aligned}
$$

This means that $L_{\sigma}^{\lambda}[a, b] \supset L_{\sigma}^{\lambda}[a, 0] \cap L_{\sigma}^{\lambda}[0, b]$ holds. The second part is easy to see.

2) If we let $\delta_{k} \equiv h$, it is easy to see

$$
L_{\sigma}^{\lambda}[a, b] \subset \cap_{k} \operatorname{Lip}_{[a, b]}^{\lambda}(k+\sigma) .
$$

On the other hand, we have

$$
\begin{aligned}
& \sum_{k}\left|f\left(k+\sigma+\delta_{k}\right)-f(k+\sigma)\right| \\
& \quad \leq \sum_{k}\|f\|_{\operatorname{Lip}_{[a, b]}^{\lambda}(k+\sigma)}\left|\delta_{k}\right|^{\lambda} \\
& \quad \leq\left(\sum_{k}\|f\|_{\operatorname{Lip}_{[a, b]}^{\lambda}(k+\sigma)}\right) \sup _{k}\left|\delta_{k}\right|^{\lambda} .
\end{aligned}
$$

This implies that the first inclusion holds.
3) Due to the Lagrange mean value formula, there exists a number sequence $\left\{\theta_{k, \sigma}\right\}_{k} \subset(0,1)$ such that

$$
\begin{aligned}
& \sum_{k}\left|f\left(k+\sigma+\delta_{k}\right)-f(k+\sigma)\right| \\
& \quad \leq \sum_{k}\left|f^{\prime}\left(k+\sigma+\theta_{k}, \sigma \delta_{k}\right)\right|\left|\delta_{k}\right| \\
& \quad \leq\left(\sum_{k} \sup _{k+\sigma+[a, b]}\left|f^{\prime}(t)\right|\right) \sup _{k}\left|\delta_{k}\right| .
\end{aligned}
$$

We now show a theorem which can lead to an algorithm to estimate the $\delta_{\varphi}$.

Theorem 2: Suppose $\varphi(t)$ is an orthogonal continuous scaling function of MRA $\left\{V_{m}\right\}_{m}$ with

1) $|\varphi(t)|=O\left(1 /|t|^{1+\varepsilon}\right)$ for some $\varepsilon>0$,

2) $\hat{\varphi}^{*}(\omega) \neq 0$,

3) $\varphi(t) \in L_{0}^{\lambda}[a, b]$.

Then for any $\left\{\delta_{k}\right\}_{k} \subset\left[-\delta_{\varphi}, \delta_{\varphi}\right] \cap[a, b]$, there exists a sequence $\left\{S_{k}(s)\right\}_{k}$ biorthogonal to $\left\{q_{\varphi /\|\varphi\|}\left(s, k+\delta_{k}\right)\right\}_{k}$ in $V_{0}$ such that (4) holds if

$$
\delta_{\varphi}<\left(\|\varphi\|_{L_{0}^{\lambda}[a, b]}^{-1}\left\|\hat{\varphi}^{*}(\omega)\right\|_{0}\right)^{1 / \lambda} .
$$

Proof: Let $g(t)=\varphi(t) /\|\varphi\|$. Then, $g(t)$ is an orthonormal continuous scaling function which satisfies the above three assumptions in Theorem 2. Following the proof of Theorem 1, we only need to show that for any $\left\{\delta_{k}\right\}_{k} \subset\left[-\delta_{\varphi}, \delta_{\varphi}\right] \cap[a, b]$ and any $\left\{c_{k}\right\}_{k} \in l^{2}$

$$
\begin{aligned}
\sum_{\alpha}\left|g\left(\alpha+\delta_{k}\right)-g(\alpha)\right| & \sum_{\beta}\left|g\left(\beta+\delta_{\beta+k-\alpha}\right)-g(\beta)\right| \\
& \cdot \sum_{k}\left|c_{k}\right|^{2} \leq \theta\left\|\sum_{k} c_{k} q_{g}(s, k)\right\|^{2} .
\end{aligned}
$$

Due to $g(t) \in L_{0}^{\lambda}[a, b]$, we have

$$
\begin{aligned}
& \sum_{\alpha}\left|g\left(\alpha+\delta_{k}\right)-g(\alpha)\right| \sum_{\beta}\left|g\left(\beta+\delta_{\beta+k-\alpha}\right)-g(\beta)\right| \\
& \quad \leq\left(\|g\|_{L_{0}^{\lambda}[a, b]}\right)^{2} \sup _{k}\left|\delta_{k}\right|^{\lambda} \sup _{k}\left|\delta_{\beta+k-\alpha}\right|^{\lambda} \\
& \quad \leq\left(\delta_{\varphi}^{\lambda}\|\varphi\|_{L_{0}^{\lambda}[a, b]} /\|\varphi\|\right)^{2} .
\end{aligned}
$$

By the way, we also have

$$
\begin{aligned}
& \left\|\sum_{k} c_{k} q_{g}(s, k)\right\|^{2} \\
& \quad=\frac{1}{2 \pi}\left\|\sum_{k} c_{k} \hat{q}_{g}(\omega, k)\right\|^{2} \\
& \quad=\frac{1}{2 \pi}\left\|\sum_{k} c_{k} \hat{g}(\omega) \sum_{n} e^{-i n \omega} g(k-n)\right\|^{2} \\
& \quad=\frac{1}{2 \pi}\left\|\sum_{k} c_{k} \hat{g}^{*}(\omega) \hat{g}(\omega) e^{-i k w}\right\|^{2}\left\|^{\wedge}\right\|^{2} \\
& \quad \geq \frac{1}{2 \pi}\left\|\hat{g}^{*}(\omega)\right\|_{0}^{2} \|\left[\sum_{k} c_{k} g(\cdot-k) \|^{(\omega)}\right.
\end{aligned}
$$




$$
\begin{aligned}
& =\left\|\hat{g}^{*}(\omega)\right\|_{0}^{2}\left\|\sum_{k} c_{k} g(s-k)\right\|^{2} \\
& =\left\|\hat{\varphi}^{*}(\omega)\right\|_{0}^{2} \sum_{k}\left|c_{k}\right|^{2} /\|\varphi\|^{2}
\end{aligned}
$$

where (23) and (27) are due to Parseval identity, and (28) is due to the orthonormality of $g(t)$. Following (20), (22), and (28), we only need to show

$$
\left(\delta_{\varphi}^{\lambda}\|\varphi\|_{L_{0}^{\lambda}[a, b]} /\|\varphi\|\right)^{2}<\left(\left\|\hat{\varphi}^{*}(\omega)\right\|_{0} /\|\varphi\|\right)^{2} .
$$

However, (19) exactly implies (29).

\section{Remark 1:}

1) $\|\varphi\|_{L_{0}^{\lambda}[a, b]}$ in (19) can be 0 . In that case, the righthand side of (19) is $\infty$. Then, Theorem 2 holds for any $\left\{\delta_{k}\right\}_{k} \subset[a, b]$.

2) Referring to Section IV, we will find $\hat{\varphi}^{*}(\omega) \in \operatorname{Lip}^{\varepsilon}$. Hence

$$
\left\|\hat{\varphi}^{*}(\omega)\right\|_{0}=\inf \left|\hat{\varphi}^{*}(\omega)\right|>0
$$

and (19) is, in fact,

$$
\delta_{\varphi}<\left(\|\varphi\|_{L_{0}^{\lambda}[a, b]}^{-1} \inf \left|\hat{\varphi}^{*}(\omega)\right|\right)^{1 / \lambda} .
$$

3) For cardinal orthonormal scaling function (see [1], [17]) $\hat{\varphi}^{*}(\omega)=1$ holds.

4) From Proposition 1, we know that $L_{0}^{\lambda}[a, b]$ is big enough. In fact, we can verify that spline, Daubechies scaling function, and Meyer scaling function are all included in it. And in a practical case we will often find $[a, b]=[-1,1],[-1,0]$, or $[0,1]$.

For a sequence $\left\{\delta_{k}\right\}_{k} \subset[a, b]$, we wish to know if we can reconstruct the original signal $f(t)$ from the sampled values $\left\{f\left(k+\delta_{k}\right)\right\}_{k}$. Of course, we can verify the conditions in Theorem 2, but the following method is simpler and more convenient, because it has less restrictive constraints than Theorem 2.

Corollary 1: In Theorem 2, if 3) is replaced by "For a sequence $\left\{\delta_{k}\right\}_{k}$, there is a constant $C_{\varphi}>0$, such that for any mapping $\tau: Z \rightarrow Z$ ( $Z$ is the integer set)

$$
\sum_{k}\left|\varphi\left(k+\delta_{\tau(k)}\right)-\varphi(k)\right| \leq C_{\varphi} \sup _{k}\left|\delta_{k}\right|^{\lambda}
$$

for some $\lambda>0$,"

then there is a sequence $\left\{S_{k}(s)\right\}_{k}$ biorthogonal to $\left\{q_{\varphi /\|\varphi\|}\left(s, k+\delta_{k}\right)\right\}_{k}$ in $V_{0}$ such that (4) holds if

$$
\sup _{k}\left|\delta_{k}\right|<\left(C_{\varphi}^{-1} \inf \left|\hat{\varphi}^{*}(\omega)\right|\right)^{1 / \lambda} .
$$

Proof: Let $g(t)=\varphi(t) /\|\varphi\|$. Then (22) becomes

$$
\begin{aligned}
\sum_{\alpha}\left|g\left(\alpha+\delta_{k}\right)-g(\alpha)\right| \sum_{\beta}\left|g\left(\beta+\delta_{\beta+k-\alpha}\right)-g(\beta)\right| \\
\leq\left(C_{\varphi} \sup \left|\delta_{k}\right|^{\lambda} /\|\varphi\|\right)^{2} .
\end{aligned}
$$

Referring to the proof of Theorem 2 and 2) of Remark 1, we only need $\sup _{k}\left|\delta_{k}\right|<\left(C_{\varphi}^{-1} \inf \left|\hat{\varphi}^{*}(\omega)\right|\right)^{1 / \lambda}$.
It seems that we can establish an algorithm for general wavelet subspaces by orthonormalizing the scaling function (refer to [1], [15], and [17]), i.e., taking $g(t)$ such as $\hat{g}(\omega)=$ $\hat{\varphi}(\omega) G_{\varphi}^{-1}(\omega)$. But obtaining $g(t)$ and calculating $\|g\|_{L_{0}^{\lambda}[a, b]}$ from $\varphi(t)$ involves convolution or the fast Fourier transform (FFT) to be undertaken twice. This is inconvenient, so we should find other proper ways to estimate $\delta_{\varphi}$ directly. First, we extend the theorem and algorithm to those for the sampling in biorthogonal wavelet subspaces, then we deduce the results for the sampling in general of wavelet subspaces.

\section{IrRegular Sampling Theorem and Algorithm FOR BIORTHOGONAL WAVELET SUBSPACES}

Theorem 3: Suppose $\{\varphi(t), \widetilde{\varphi}(t)\}$ is a biorthogonal continuous scaling function pair of the MRA pair $\left\{V_{m}, \tilde{V}_{m}\right\}_{m}$ (with $V_{0}=\tilde{V}_{0}$ ), which satisfies

1) $\varphi(t) \in L^{\infty}(R)$, and $|\tilde{\varphi}(t)| \leq O\left(1 /|t|^{1+\varepsilon}\right)$ for some

$\varepsilon>0$

2) $\hat{\tilde{\varphi}}^{*}(\omega) \neq 0$.

Then there exists a $\delta_{\varphi, \tilde{\varphi}} \in(0,1]$, such that for any $\left\{\delta_{k}\right\}_{k} \subset$ $\left[-\delta_{\varphi, \tilde{\varphi}}, \delta_{\varphi}, \tilde{\varphi}\right]$, there is a sequence $\left\{S_{k}(s)\right\}_{k}$ biorthogonal to $\left\{q_{\varphi}, \tilde{\varphi}\left(s, k+\delta_{k}\right)\right\}_{k}$ in $V_{0}$ such that (4) holds.

We need two lemmas for the proof of the theorem.

Lemma 2: Under the same assumption as Theorem 3, $\left\{q_{\varphi}, \tilde{\varphi}(s, k)\right\}_{k}$ is a Riesz basis of $V_{0}$.

Proof: It is easy to see that $q_{\varphi}, \tilde{\varphi}(s, t)$ is well-defined and $\left\{q_{\varphi}, \tilde{\varphi}(s, k)\right\}_{k} \subset V_{0}$. Let $T$ be the linear operator on $V_{0}$ that takes

$$
\sum_{k} c_{k} \varphi(t-k)
$$

into

$$
\sum_{k} c_{k} q_{\varphi, \tilde{\varphi}}(s, k)
$$

for any $\left\{c_{k}\right\}_{k} \in l^{2}$. Since

$$
\begin{aligned}
& \left\|\sum_{k} c_{k} q_{\varphi, \tilde{\varphi}}(s, k)\right\|^{2} \\
& \quad=\frac{1}{2 \pi}\left\|\sum_{k} c_{k} \hat{q}_{\varphi, \tilde{\varphi}}(\omega, k)\right\|^{2} \\
& =\frac{1}{2 \pi}\left\|\sum_{k} c_{k} \hat{\tilde{\varphi}}^{*}(\omega) \hat{\varphi}(\omega) e^{-i k \omega}\right\|^{2} \\
& =\frac{1}{2 \pi}\left\|\hat{\tilde{\varphi}}^{*}(\omega) G_{\varphi}(\omega) \sum_{k} c_{k} e^{-i k \omega}\right\|_{L^{2}[0,2 \pi]}^{2}
\end{aligned}
$$

we obtain

$$
\begin{aligned}
\left\|\hat{\tilde{\varphi}}^{*}(\omega) G_{\varphi}(\omega)\right\|_{0}^{2} \sum_{k}\left|c_{k}\right|^{2} & \leq\left\|\sum_{k} c_{k} q_{\varphi, \tilde{\varphi}}(s, k)\right\|^{2} \\
& \leq\left\|\hat{\tilde{\varphi}}^{*}(\omega) G_{\varphi}(\omega)\right\|_{\infty}^{2} \sum_{k}\left|c_{k}\right|^{2} .
\end{aligned}
$$


On the other hand, from

$$
\begin{aligned}
\left\|\sum_{k} c_{k} \varphi(s-k)\right\|^{2} & =\frac{1}{2 \pi}\left\|\hat{\varphi}(\omega) \sum_{k} c_{k} e^{-i k \omega}\right\|^{2} \\
& =\frac{1}{2 \pi}\left\|G_{\varphi}(\omega) \sum_{k} c_{k} e^{-i k \omega}\right\|_{L^{2}[0,2 \pi]}^{2}
\end{aligned}
$$

we can deduce that

$$
\begin{aligned}
\left\|G_{\varphi}(\omega)\right\|_{0}^{2} \sum_{k}\left|c_{k}\right|^{2} & \leq\left\|\sum_{k} c_{k} \varphi(s-k)\right\|^{2} \\
& \leq\left\|G_{\varphi}(\omega)\right\|_{\infty}^{2} \sum_{k}\left|c_{k}\right|^{2} .
\end{aligned}
$$

Since $\hat{\tilde{\varphi}}^{*}(\omega)$ is a continuous nonzero $2 \pi$-periodic function, we derive

$$
0<\inf \left|\hat{\tilde{\varphi}}^{*}(\omega)\right| \leq \sup \left|\hat{\tilde{\varphi}}^{*}(\omega)\right|<\infty .
$$

It is well known (see [1], [15], and [17]) that

$$
0<\left\|G_{\varphi}(\omega)\right\|_{0} \leq\left\|G_{\varphi}(\omega)\right\|_{\infty}<\infty .
$$

From (34), (35), (37), and (38), we can now conclude

$$
0<\frac{\left\|\hat{\tilde{\varphi}}^{*}(\omega) G_{\varphi}(\omega)\right\|_{0}}{\left\|G_{\varphi}(\omega)\right\|_{\infty}} \leq\|T\| \leq \frac{\left\|\hat{\tilde{\varphi}}^{*}(\omega) G_{\varphi}(\omega)\right\|_{\infty}}{\left\|G_{\varphi}(\omega)\right\|_{0}}<\infty .
$$

Moreover, for any $g(t) \in V_{0}$, take $f(t)$ such that $\hat{f}(\omega)=$ $\hat{g}(\omega) / \hat{\varphi}^{*}(\omega)$. Then it is easy to show that $f(t) \in V_{0}$ and $T(f)=g$. Therefore, $T$ is a linear mapping which is continuous, one to one and onto, i.e., $\left\{q_{\varphi}, \tilde{\varphi}(s, k)\right\}_{k}$ is a Riesz basis of $V_{0}$.

Lemma 3: Suppose the biorthogonal continuous scaling function pair $\{\varphi(t), \tilde{\varphi}(t)\}$ of the MRA pair $\left\{V_{m}, \tilde{V}_{m}\right\}_{m}$ (with $V_{0}=\tilde{V}_{0}$ ) satisfies $\varphi(t) \in L^{\infty}(R),|\tilde{\varphi}(t)| \leq O\left(1 /|t|^{1+\varepsilon}\right.$ ) for some $\varepsilon>0$. Then

$$
f(t)=\int_{R} f(s) q_{\varphi, \tilde{\varphi}}(s, t) d s, \quad \text { for } f \in V_{0} .
$$

Proof: $\varphi(s) \in L^{\infty}(R)$ implies that $|\varphi(s-n)|$ is uniformly bounded with respect to a.e. $s$ in $R .|\tilde{\varphi}(t)| \leq$ $O\left(1 /|t|^{1+\varepsilon}\right)$ and the continuity of $\tilde{\varphi}(t)$ imply that

$$
\sum_{n}|\tilde{\varphi}(t-n)|
$$

is uniformly convergent with respect to $s$ in $R$. Therefore,

$$
\sum_{n} \varphi(s-n) \tilde{\varphi}(t-n)
$$

converges uniformly with respect to a.e. $s$ in $R$. For $f \in V_{0}=$ $\tilde{V}_{0}$, let

$$
f(t)=\sum_{k} c_{k} \tilde{\varphi}(t-k)
$$

Then

$$
\begin{aligned}
& \int_{R} f(s) q_{\varphi, \tilde{\varphi}}(s, t) d s \\
& \quad=\sum_{n} \tilde{\varphi}(t-n) \sum_{k} c_{k} \int_{R} \varphi(s-n) \tilde{\varphi}(s-k) d s \\
& \quad=f(t) .
\end{aligned}
$$

Proof of Theorem: If we can show that $\left\{q_{\varphi}, \tilde{\varphi}\left(s, k+\delta_{k}\right)\right\}_{k}$ is a Riesz basis in $V_{0}$, then there is a sequence $\left\{S_{k}(s)\right\}_{k}$ biorthogonal to $\left\{q_{\varphi}, \tilde{\varphi}\left(s, k+\delta_{k}\right)\right\}_{k}$ in $V_{0}$, such that

$$
f(s)=\sum_{k} S_{k}(s) \int_{R} f(s) q_{\varphi, \tilde{\varphi}}\left(s, k+\delta_{k}\right) d s, \quad f \in V_{0} .
$$

Following Lemma 3, it is easy to see that (4) holds. However, Lemma 2 tells us that $\left\{q_{\varphi, \tilde{\varphi}}(s, k)\right\}_{k}$ is a Riesz basis in $V_{0}$. So we only need to find a $\delta_{\varphi, \tilde{\varphi}} \in(0,1]$, such that for any $\left\{\delta_{k}\right\}_{k} \subset\left[-\delta_{\varphi, \tilde{\varphi}}, \delta_{\varphi, \tilde{\varphi}}\right]$, there is a $\theta \in[0,1)$ such that for any $\left\{c_{k}\right\}_{k} \in l^{2}$

$$
\begin{aligned}
&\left\|\sum_{k} c_{k} q_{\varphi, \tilde{\varphi}}\left(s, k+\delta_{k}\right)-\sum_{k} c_{k} q_{\varphi, \tilde{\varphi}}(s, k)\right\|^{2} \\
& \leq \theta\left\|\sum_{k} c_{k} q_{\varphi, \tilde{\varphi}}(s, k)\right\|^{2}
\end{aligned}
$$

In order to show (44), let

$$
\begin{aligned}
\Delta & =\left\|\sum_{k} c_{k} q_{\varphi, \tilde{\varphi}}\left(s, k+\delta_{k}\right)-\sum_{k} c_{k} q_{\varphi, \tilde{\varphi}}(s, k)\right\|^{2} \\
& =\| \sum_{n}\left(\sum _ { k } c _ { k } \left(\tilde{\varphi}\left(k+\delta_{k}-n\right)\right.\right.
\end{aligned}
$$$$
-\tilde{\varphi}(k-n))) \varphi(s-n) \|^{2}
$$$$
=\frac{1}{2 \pi} \| \sum_{n}\left(\sum _ { k } c _ { k } \left(\tilde{\varphi}\left(k+\delta_{k}-n\right)\right.\right.
$$$$
-\tilde{\varphi}(k-n))) \hat{\varphi}(\omega) e^{-i n \omega} \|^{2}
$$$$
=\frac{1}{2 \pi} \| G_{\varphi}(\omega) \sum_{n}\left(\sum _ { k } c _ { k } \left(\tilde{\varphi}\left(k+\delta_{k}-n\right)\right.\right.
$$

$$
-\tilde{\varphi}(k-n))) e^{-i n \omega} \|_{L^{2}[0,2 \pi]}^{2}
$$

$$
\leq\left\|G_{\varphi}(\omega)\right\|_{\infty}^{2} \sum_{n} \mid \sum_{k} c_{k}\left(\tilde{\varphi}\left(k+\delta_{k}-n\right)\right.
$$

$$
\begin{gathered}
-\tilde{\varphi}(k-n))\left.\right|^{2} \\
=\left\|G_{\varphi}(\omega)\right\|_{\infty}^{2} \sum_{n} \sum_{k, l}\left(\tilde{\varphi}\left(k+\delta_{k}-n\right)-\tilde{\varphi}(k-n)\right) \\
\cdot\left(\tilde{\varphi}\left(l+\delta_{l}-n\right)-\tilde{\varphi}(l-n)\right) c_{k} c_{l} \\
=\left\|G_{\varphi}(\omega)\right\|_{\infty}^{2} \sum_{k, l}\left(\sum_{n}\left(\tilde{\varphi}\left(k+\delta_{k}-n\right)-\tilde{\varphi}(k-n)\right)\right.
\end{gathered}
$$

Denote

$$
\left.\cdot\left(\tilde{\varphi}\left(l+\delta_{l}-n\right)-\tilde{\varphi}(l-n)\right)\right) c_{k} c_{l} .
$$

$b_{k, l}=\sum_{n}\left(\tilde{\varphi}\left(k+\delta_{k}-n\right)-\tilde{\varphi}(k-n)\right)\left(\tilde{\varphi}\left(l+\delta_{l}-n\right)-\tilde{\varphi}(l-n)\right)$. 
Then $b_{k, l}=b_{l, k}$ and

$$
\begin{aligned}
\Delta & \leq\left\|G_{\varphi}(\omega)\right\|_{\infty}^{2} \sum_{k, l} b_{k, l} c_{k} c_{l} \\
& \leq\left\|G_{\varphi}(\omega)\right\|_{\infty}^{2}\left(\sup _{k} \sum_{l}\left|b_{k, l}\right|\right) \sum_{k} c_{k}^{2} .
\end{aligned}
$$

By the way, we have

$$
\begin{aligned}
\sup _{k} \sum_{l}\left|b_{k, l}\right| \leq & \sup _{k} \sum_{l} \sum_{n}\left|\tilde{\varphi}\left(k+\delta_{k}-n\right)-\tilde{\varphi}(k-n)\right| \\
& \cdot\left|\tilde{\varphi}\left(l+\delta_{l}-n\right)-\tilde{\varphi}(l-n)\right| \\
\leq & \sup _{k} \sum_{\alpha}\left|\tilde{\varphi}\left(\alpha+\delta_{k}\right)-\tilde{\varphi}(\alpha)\right| \\
& \cdot \sum_{\beta}\left|\tilde{\varphi}\left(\beta+\delta_{\beta+k-\alpha}\right)-\tilde{\varphi}(\beta)\right|
\end{aligned}
$$

where (46) is due to the index transform $\alpha=k-n$ and $\beta=l-n$. Following the proof of Theorem 1, the constraint $|\tilde{\varphi}(t)| \leq O\left(1 /|t|^{1+\varepsilon}\right.$ ) for some $\varepsilon>0$ and the continuity of $\tilde{\varphi}(t)$, we can conclude that there is a $\delta_{\varphi, \tilde{\varphi}} \in(0,1]$, such that for any $\left\{\delta_{k}\right\}_{k} \subset\left[-\delta_{\varphi, \tilde{\varphi}}, \delta_{\varphi, \tilde{\varphi}]}\right.$

$$
\begin{array}{r}
\sup _{k} \sum_{\alpha}\left|\tilde{\varphi}\left(\alpha+\delta_{k}\right)-\tilde{\varphi}(\alpha)\right| \sum_{\beta}\left|\tilde{\varphi}\left(\beta+\delta_{\beta+k-\alpha}\right)-\tilde{\varphi}(\beta)\right| \\
\leq \frac{\theta\left\|\hat{\tilde{\varphi}}^{*}(\omega) G_{\varphi}(\omega)\right\|_{0}^{2}}{\left\|G_{\varphi}(\omega)\right\|_{\infty}^{2}}
\end{array}
$$

From (45)-(47), it was followed that

$$
\Delta \leq \theta\left\|\hat{\tilde{\varphi}}^{*}(\omega) G_{\varphi}(\omega)\right\|_{0}^{2} \sum_{k} c_{k}^{2}
$$

holds. Then from (35), we conclude that (44) holds.

Based on the theorem, we can now provide an algorithm for sampling in biorthogonal wavelet subspaces to calculate the $\delta_{\varphi, \tilde{\varphi}}$ from the biorthogonal scaling function pair $\{\varphi(t), \tilde{\varphi}(t)\}$.

Theorem 4: Suppose $\{\varphi(t), \tilde{\varphi}(t)\}$ is a continuous biorthogonal scaling function pair of the MRA pair $\left\{V_{m}, \tilde{V}_{m}\right\}_{m}$ (with $V_{0}=\tilde{V}_{0}$ ), which satisfies

1) $\varphi(t) \in L^{\infty}(R)$, and $|\tilde{\varphi}(t)|=O\left(1 /|t|^{1+\varepsilon}\right)$ for some $\varepsilon>0$

2) $\hat{\tilde{\varphi}}^{*}(\omega) \neq 0$,

3) $\tilde{\varphi}(t) \in L_{0}^{\lambda}[a, b]$.

Then for any $\left\{\delta_{k}\right\}_{k} \subset\left[-\delta_{\varphi, \tilde{\varphi}}, \delta_{\varphi}, \tilde{\varphi}\right] \cap[a, b]$, there exists a sequence $\left\{S_{k}(t)\right\}_{k}$ biorthogonal to $\left\{q_{\varphi}, \tilde{\varphi}\left(s, k+\delta_{k}\right)\right\}_{k}$ in $V_{0}$ such that (4) holds if

$$
\delta_{\varphi, \tilde{\varphi}}<\left(\frac{\left\|\hat{\tilde{\varphi}}^{*}(\omega) G_{\varphi}(\omega)\right\|_{0}}{\left\|G_{\varphi}(\omega)\right\|_{\infty}\|\tilde{\varphi}\|_{L_{0}^{\lambda}[a, b]}}\right)^{1 / \lambda} .
$$

Proof: Following the proof of Theorem 3, we only need to show that for any $\left\{\delta_{k}\right\}_{k} \subset\left[-\delta_{\varphi, \tilde{\varphi}}, \delta_{\varphi}, \tilde{\varphi}\right] \cap[a, b]$

$$
\begin{aligned}
\sum_{\alpha}\left|\tilde{\varphi}\left(\alpha+\delta_{k}\right)-\tilde{\varphi}(\alpha)\right| \sum_{\beta} \mid \tilde{\varphi}(\beta & \left.+\delta_{\beta+k-\alpha}\right)-\tilde{\varphi}(\beta) \mid \\
\leq & \frac{\theta\left\|\hat{\tilde{\varphi}}^{*}(\omega) G_{\varphi}(\omega)\right\|_{0}^{2}}{\left\|G_{\varphi}(\omega)\right\|_{\infty}^{2}} .
\end{aligned}
$$

Due to $\tilde{\varphi}(t) \in L_{0}^{\lambda}[a, b]$, we know

$$
\begin{aligned}
\sum_{\alpha}\left|\tilde{\varphi}\left(\alpha+\delta_{k}\right)-\tilde{\varphi}(\alpha)\right| \sum_{\beta} \mid \tilde{\varphi}(\beta & \left.+\delta_{\beta+k-\alpha}\right)-\tilde{\varphi}(\beta) \mid \\
& \leq\left(\delta_{\varphi, \tilde{\varphi}}^{\lambda}\|\tilde{\varphi}\|_{L_{0}^{\lambda}[a, b]}\right)^{2} .
\end{aligned}
$$

Equations (49) and (50) imply that we only need

$$
\left(\delta_{\varphi, \tilde{\varphi}}^{\lambda}\|\tilde{\varphi}\|_{L_{0}^{\lambda}[a, b]}\right)^{2}<\frac{\left\|\hat{\tilde{\varphi}}^{*}(\omega) G_{\varphi}(\omega)\right\|_{0}^{2}}{\left\|G_{\varphi}(\omega)\right\|_{\infty}^{2}} .
$$

This is exactly implied by (48).

Remark 2:

1) As the sampling in orthogonal wavelet subspaces, $\|\tilde{\varphi}(t)\|_{L_{0}^{\lambda}[a, b]}$ in (48) can be zero. Then, Theorem 4 holds for any $\left\{\delta_{k}\right\}_{k} \subset[a, b]$.

2) Since $\hat{\tilde{\varphi}}^{*}(\omega) \in \operatorname{Lip}^{\varepsilon}$ (refer to Section IV) and $\left\|G_{\varphi}(\omega)\right\|_{0}>0$, we know that $\left\|\hat{\tilde{\varphi}}^{*}(\omega) G_{\varphi}(\omega)\right\|_{0}>0$.

3) In the orthogonal case, $G_{\varphi}(\omega)$ is constant a.e. $\omega$ and $\tilde{\varphi}(t)=\varphi(t) /\|\varphi\|^{2}$. Therefore, (48) is the same as (19).

4) From the proof of the theorem, we also find that $\varphi(t)$ does not need to be continuous.

There is another simple way to verify the conditions for a given sampling in biorthogonal wavelet subspace.

Corollary 2: In Theorem 4, if 3) is replaced by "For a sequence $\left\{\delta_{k}\right\}_{k}$ there is a constant $C_{\tilde{\varphi}}>0$ such that for any mapping $\tau: Z \rightarrow Z$ ( $Z$ is the integer set)

$$
\sum_{k}\left|\tilde{\varphi}\left(k+\delta_{\tau(k)}\right)-\tilde{\varphi}(k)\right| \leq C_{\tilde{\varphi}} \sup \left|\delta_{k}\right|^{\lambda}
$$

for some $\lambda>0$,"

then there is a sequence $\left\{S_{k}(t)\right\}_{k}$ biorthogonal to $\left\{q_{\varphi}, \tilde{\varphi}(s, k)\right\}_{k}$ in $V_{0}$ such that (4) holds if

$$
\sup _{k}\left|\delta_{k}\right|<\left(\frac{\left\|\hat{\tilde{\varphi}}^{*}(\omega) G_{\varphi}(\omega)\right\|_{0}}{C_{\tilde{\varphi}}\left\|G_{\varphi}(\omega)\right\|_{\infty}}\right)^{1 / \lambda} .
$$

Now following the results for biorthogonal wavelet subspaces, we can show an irregular sampling theorem and provide an algorithm for the sampling in general wavelet subspaces.

\section{IRREgular SAMPling THEOREM AND Algorithm FOR General WAVElEt SubSPaCes}

Theorem 5: Suppose the continuous scaling function $\varphi(t)$ of MRA $\left\{V_{m}\right\}_{m}$ satisfies:

1) $|\varphi(t)| \leq O\left(1 /|t|^{1+\varepsilon}\right)$ for some $\varepsilon>0$,

2) $\hat{\varphi}^{*}(\omega) \neq 0$.

Then there is a $\delta_{\varphi} \in(0,1]$, such that for any $\left\{\delta_{k}\right\}_{k} \subset$ $\left[-\delta_{\varphi}, \delta_{\varphi}\right]$, there is a sequence $\left\{S_{k}(t)\right\}_{k} \subset V_{0}$ such that (9) holds.

In order to show the theorem, we need two lemmas.

Lemma 4: Suppose the continuous scaling function $\varphi(t)$ of MRA $\left\{V_{m}\right\}_{m}$ satisfies the same constraints as Theorem 5 . Then $\left\{q_{\varphi}(t, k)\right\}_{k}$ is a Riesz basis in $V_{0}$. Suppose $\left\{\tilde{q}_{k}(t)\right\}_{k}$ is biorthogonal to $\left\{q_{\varphi}(t, k)\right\}_{k}$. Then $\tilde{q}_{k}(t)=\tilde{q}_{0}(t-k)$ and

$$
\hat{\tilde{q}}_{0}(\omega)=\hat{\varphi}(\omega) \overline{\hat{\varphi}^{*}(\omega)} G_{\varphi}^{2}(\omega) .
$$


Proof: Referring to Lemma 2, it is easy to show that $\left\{q_{\varphi}(t, k)\right\}_{k}$ is a Riesz basis in $V_{0}$. Since $q_{\varphi}(t, k)=q_{\varphi}(t-$ $k, 0),\left\{\tilde{q}_{0}(t-k)\right\}_{k}$ is biorthogonal to $q_{\varphi}(t-k, 0)$, we have $\tilde{q}_{k}(t)=\tilde{q}_{0}(t-k)$ due to the uniqueness of $\tilde{q}_{k}(t)$ (see [5], [9], and [17]). Let

$$
G_{\varphi}(\omega)=\sum_{k} d_{k} e^{i k \omega}
$$

Then due to $|\varphi(t)| \leq O\left(1 /|t|^{1+\varepsilon}\right.$ ) (see [12] and [15])

$$
\begin{aligned}
\left|d_{k}\right|= & \left|\int_{R} \varphi(t) \varphi(t+k) d t\right| \\
\leq & \int_{|t| \leq|k| / 2}|\varphi(t) \varphi(t+k)| d t \\
& +\int_{|t| \geq|k| / 2} \varphi(t)|\varphi(t+k)| d t \\
\leq & O\left(1 /|k|^{1+\varepsilon}\right) \int_{|t| \leq|k| / 2}|\varphi(t)| d t \\
& +O\left(1 /|k|^{1+\varepsilon}\right) \int_{|t| \geq|k| / 2}|\varphi(t+k)| d t \\
\leq & O\left(1 /|k|^{1+\varepsilon}\right) .
\end{aligned}
$$

Equation (52) implies $\left\{d_{k}\right\}_{k} \in l^{1}$. Of course, we also have $\{\varphi(k)\}_{k} \in l^{1}$. Let

$$
1 \overline{\hat{\varphi}}^{*} G_{\varphi}^{2}(\omega)=\sum_{k} c_{k} e^{i k \omega}
$$

Since $\hat{\varphi}^{*}(\omega)$ and $G_{\varphi}^{2}(\omega)$ are continuous (refer to the proof of Lemma 5), we have $\left\{c_{k}\right\}_{k} \in l^{1} \subset l^{2}$ due to Wiener-Lévy Theorem (see [6]). Since

$$
\left\|\hat{\varphi}(\omega) e^{-i l \omega} \overline{\hat{\varphi}^{*}(\omega)} G_{\varphi}^{2}(\omega)\right\| \leq O(\|\varphi\|)
$$

we can take the inverse Fourier transform of

$$
\hat{\varphi}(\omega) e^{-i l \omega} \overline{\overline{\hat{\varphi}^{*}}(\omega)} G_{\varphi}^{2}(\omega)
$$

in $L^{2}(R)$ as denoted by

$$
\left[\hat{\varphi}(\omega) e^{-i l \omega} \overline{\hat{\varphi}^{*}(\omega)} G_{\varphi}^{2}(\omega)\right]^{\vee}(s)
$$

(refer to the Introduction). Now we obtain

$$
\left[\frac{\hat{\varphi}(\omega)}{\overline{\hat{\varphi}^{*}(\omega)} G_{\varphi}^{2}(\omega)} e^{-i l \omega}\right]^{\vee}(s)=\sum_{k} c_{k} \varphi(s-k-l) .
$$

Equation (53) implies $\left[\left(\hat{\varphi}(\omega) \overline{\hat{\varphi}^{*}(\omega)} G_{\varphi}^{2}(\omega)\right) e^{-i l \omega}\right]^{\vee}(s) \in V_{0}$. By the way, we also have

$$
\begin{aligned}
& \int_{R} q_{\varphi}(s, k) \overline{\left[\frac{\hat{\varphi}(\omega)}{\overline{\hat{\varphi}^{*}(\omega)} G_{\varphi}^{2}(\omega)} e^{-i l \omega}\right]^{\vee}(s)} d s \\
& \quad=\frac{1}{2 \pi} \int_{R} \hat{q}_{\varphi}(\omega, k) \frac{\overline{\hat{\varphi}(\omega)}}{\hat{\varphi}^{*}(\omega) G_{\varphi}^{2}(\omega)} e^{i l \omega} d \omega \\
& \quad=\frac{1}{2 \pi} \int_{R} \hat{\varphi}^{*}(\omega) \hat{\varphi}(\omega) e^{-i k \omega} \frac{\overline{\hat{\varphi}(\omega)}}{\hat{\varphi}^{*}(\omega) G_{\varphi}^{2}(\omega)} e^{i l \omega} d \omega \\
& \quad=\frac{1}{2 \pi} \int_{R} \frac{|\hat{\varphi}(\omega)|^{2}}{G_{\varphi}^{2}(\omega)} e^{-i(k-l) \omega} d \omega
\end{aligned}
$$

$$
\begin{aligned}
& =\frac{1}{2 \pi} \int_{0}^{2 \pi} \frac{\sum_{k}|\hat{\varphi}(\omega+2 k \pi)|^{2}}{G_{\varphi}^{2}(\omega)} e^{-i(k-l) \omega} d \omega \\
& =\delta_{k l l} .
\end{aligned}
$$

Equation (56) implies that $\left\{\left[\hat{\varphi}(\omega) e^{-i k \omega} / \overline{\hat{\varphi}^{*}(\omega)} G_{\varphi}^{2}(\omega)\right]^{\vee}(s)\right\}_{k}$ is biorthogonal to $\left\{q_{\varphi}(s, k)\right\}_{k}$. Hence

$$
\tilde{q}_{0}(s-k)=\left[\hat{\varphi}(\omega) e^{-i k \omega} \overline{\hat{\varphi}^{*}(\omega)} G_{\varphi}^{2}(\omega)\right]^{\vee}(s)
$$

due to the uniqueness of $\left\{\tilde{q}_{0}(s-k)\right\}_{k}$ in $V_{0}$. It deduces that

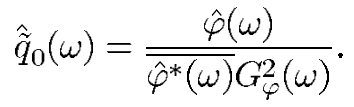

Lemma 5: Suppose the continuous scaling function $\varphi(t)$ of MRA $\left\{V_{m}\right\}_{m}$ satisfies the same constraints as Theorem 5 . Then $\tilde{q}_{0}(t)$ defined in Lemma 4 is continuous and $\left|\tilde{q}_{0}(t)\right| \leq$ $O\left(1 /|t|^{\varepsilon}\right)$.

Proof: Suppose

$$
1 \longdiv { \hat { \varphi } ^ { * } ( \omega ) } G _ { \varphi } ^ { 2 } ( \omega ) = \sum _ { k } c _ { k } e ^ { i k \omega } .
$$

Then

$$
\tilde{q}_{0}(t)=\sum_{k} c_{k} \varphi(t-k) .
$$

Due to $|\varphi(t)| \leq O\left(1 /|t|^{1+\varepsilon}\right)$, we obtain

$$
\begin{aligned}
\left|\sum_{k \geq n} \varphi(k) e^{i k \omega}\right| & \leq \sum_{k \geq n}|\varphi(k)| \\
& \leq O\left(\sum_{k \geq n} 1 /|k|^{1+\varepsilon}\right) \\
& \leq O\left(1 /|n|^{\varepsilon}\right) .
\end{aligned}
$$

Equation (59) implies $\hat{\varphi}^{*}(\omega) \in \operatorname{Lip}^{\varepsilon}$ (see [6]). For the same reason, (52) implies that $G_{\varphi}^{2}(\omega) \in \operatorname{Lip}^{\varepsilon}$ holds. Therefore,

$$
1 / \overline{\hat{\tilde{\varphi}}^{*}(\omega)} G_{\varphi}^{2}(\omega) \in \operatorname{Lip}^{\varepsilon} .
$$

It means $\left|c_{k}\right| \leq O\left(1 /|k|^{\varepsilon}\right.$ ) (of course, $\sup _{k}\left|c_{k}\right|<\infty$ ). Now we are to calculate (58):

$$
\begin{aligned}
& \quad\left|\tilde{q}_{0}(t)\right| \\
& \quad \leq\left|\sum_{|k| \leq|t| / 2} c_{k} \varphi(t-k)\right|+\left|\sum_{|k|>|t| / 2} c_{k} \varphi(t-k)\right| \\
& \quad \leq O\left(1 /|t|^{1+\varepsilon}\right) \sum_{|k| \leq|t| / 2}\left|c_{k}\right|+O\left(1 /|t|^{\varepsilon}\right) \sum_{|k|>|t| / 2}|\varphi(t-k)| \\
& \quad \leq O\left(1 /|t|^{1+\varepsilon}\right) O(|t|)+O\left(1 /|t|^{\varepsilon}\right) \sum_{k}|\varphi(t-k)| \\
& \quad \leq O\left(1 /|t|^{\varepsilon}\right) .
\end{aligned}
$$

The continuity of $\tilde{q}_{0}(t)$ follows because $\sum_{k} c_{k} \varphi(t-k)$ uniformly converges with respect to $t$ on any closed interval $[-N, N] \subset R$. 
Proof of Theorem: Following Lemma 4, we know that $\left\{\tilde{q}_{0}(t), q_{\varphi}(t, 0)\right\}$ is a biorthogonal scaling function pair of the MRA pair $\left\{V_{m}, V_{m}\right\}_{m}$. Following Lemma 5, we derive that $\tilde{q}_{0}(t) \in L^{\infty}(R)$ is continuous. By the way, we have

$$
\begin{aligned}
& \left|q_{\varphi}(t, 0)\right| \\
& \leq\left|\sum_{|k| \leq|t| / 2} \varphi(k) \varphi(t-k)\right|+\left|\sum_{|k|>|t| / 2} \varphi(t) \varphi(t-k)\right| \\
& \leq O\left(1 /|t|^{1+\varepsilon}\right) \sum_{|k| \leq|t| / 2}|\varphi(k)| \\
& \quad+O\left(1 /|t|^{1+\varepsilon}\right) \sum_{|k|>|t| / 2}|\varphi(t-k)| \\
& \quad \leq O\left(1 /|t|^{1+\varepsilon}\right) \sum_{k}|\varphi(k)|+O\left(1 /|t|^{1+\varepsilon}\right) \sum_{k}|\varphi(t-k)| \\
& \leq O\left(1 /|t|^{1+\varepsilon}\right) .
\end{aligned}
$$

In the meantime, $\hat{q}_{\varphi}^{*}(\omega, 0)=\hat{\varphi}^{*}(\omega) \overline{\hat{\varphi}^{*}}(\omega) \neq 0$ holds. Now we can apply Theorem 3 to the biorthogonal pair $\left\{\tilde{q}_{0}(t), q_{\varphi}(t, 0)\right\}$ to conclude the proof.

Based on the theorem, we can provide an algorithm for the sampling in general wavelet subspaces by generalizing Theorem 4.

Theorem 6: Suppose the continuous scaling function $\varphi(t)$ of MRA $\left\{V_{m}\right\}_{m}$ satisfies

1) $|\varphi(t)| \leq O\left(1 /|t|^{1+\varepsilon}\right)$ for some $\varepsilon>0$,

2) $\hat{\varphi}^{*}(\omega) \neq 0$,

3) $\varphi(t) \in L_{0}^{\lambda}[a, b]$.

Then for any $\left\{\delta_{k}\right\}_{k} \subset\left[-\delta_{\varphi}, \delta_{\varphi}\right] \cap[a, b]$, there is a sequence $\left\{S_{k}(s)\right\}_{k} \subset V_{0}$ such that (9) holds if

$$
\delta_{\varphi}<\left(\frac{\left\|\hat{\varphi}^{*}(\omega) / G_{\varphi}(\omega)\right\|_{0}\left\|\hat{\varphi}^{*}(\omega) G_{\varphi}(\omega)\right\|_{0}}{\left\|q_{\varphi}(s, 0)\right\|_{L_{0}^{\lambda}[a, b]}}\right)^{1 / \lambda} .
$$

Proof: We have, for any $\left\{\delta_{k}\right\}_{k} \subset[a, b]$

$$
\begin{aligned}
& \sum_{k}\left|q_{\varphi}\left(k+\delta_{k}, 0\right)-q_{\varphi}(k, 0)\right| \\
& \quad=\sum_{k}\left|\sum_{n} \varphi\left(k+\delta_{k}-n\right) \varphi(-n)-\sum_{n} \varphi(k-n) \varphi(-n)\right| \\
& \quad \leq \sum_{n}|\varphi(-n)| \sum_{k}\left|\varphi\left(k+\delta_{k}-n\right)-\varphi(k-n)\right| \\
& \quad=\sum_{n}|\varphi(-n)| \sum_{l}\left|\varphi\left(l+\delta_{l+n}\right)-\varphi(l)\right| \\
& \quad \leq\left.\sum_{n}|\varphi(-n)||| \varphi\right|_{L_{0}^{\lambda}[a, b]} \sup _{k}\left|\delta_{k}\right|^{\lambda} .
\end{aligned}
$$

This implies $q_{\varphi}(s, 0) \in L_{0}^{\lambda}[a, b]$. Now referring to the proof of Theorem 5, we can apply Theorem 4 to the biorthogonal pair $\left\{\tilde{q}_{0}(t), q_{\varphi}(t, 0)\right\}$. In this case, (48) becomes

$$
\delta_{\varphi}<\left(\frac{\left\|\hat{q}_{\varphi}^{*}(\omega, 0) G_{\tilde{q}_{0}}(\omega)\right\|_{0}}{\left\|G_{\tilde{q}_{0}}(\omega)\right\|_{\infty}\left\|q_{\varphi}(s, 0)\right\|_{L_{0}^{\lambda}[a, b]}}\right)^{1 / \lambda} .
$$

Since

$$
\begin{aligned}
G_{\tilde{q}_{0}}(\omega) & =\left(\sum_{k}\left|\hat{\tilde{q}}_{0}(\omega+2 k \pi)\right|^{2}\right)^{1 / 2} \\
& =\left(\sum_{k}\left|\hat{\varphi}(\omega+2 k \pi) / \overline{\hat{\varphi}^{*}(\omega)} G_{\varphi}^{2}(\omega)\right|^{2}\right)^{1 / 2} \\
& =G_{\varphi}(\omega) /\left|\overline{\hat{\varphi}^{*}(\omega)} G_{\varphi}^{2}(\omega)\right| \\
& =1 /\left|\hat{\varphi}^{*}(\omega)\right| G_{\varphi}(\omega)
\end{aligned}
$$

and

$$
\hat{q}_{\varphi}^{*}(\omega, 0)=\hat{\varphi}^{*}(\omega) \overline{\hat{\varphi}^{*}}(\omega)
$$

we have the theorem by calculating (61).

The following corollary is easily shown by referring to the proof of Corollary 2.

Corollary 3: In Theorem 6, if 3) is replaced by "For a sequence $\left\{\delta_{k}\right\}_{k}$ there is a constant $C_{\varphi}>0$ such that for any mapping $\tau: Z \rightarrow Z$ ( $Z$ is the integer set)

$$
\sum_{k}\left|\varphi\left(k+\delta_{\tau(k)}\right)-\varphi(k)\right| \leq C_{\varphi} \sup _{k}\left|\delta_{k}\right|^{\lambda}
$$

for some $\lambda>0$,"

then there is an $\left\{S_{k}(s)\right\}_{k} \subset V_{0}$ such that (4) holds if

$$
\sup _{k}\left|\delta_{k}\right|<\left(C_{\varphi}^{-1}\left\|\hat{\varphi}^{*}(\omega) / G_{\varphi}(\omega)\right\|_{0}\left\|\hat{\varphi}^{*}(\omega) G_{\varphi}(\omega)\right\|_{0}\right)^{1 / \lambda} .
$$

\section{Remark 3:}

1) The $\left\{S_{k}(s)\right\}_{k}$ in Theorems 5 and 6, and Corollary 3 are biorthogonal to $\left\{q_{\tilde{q}_{0}}(\cdot), q_{\varphi}(\cdot, 0)\left(s, k+\delta_{k}\right)\right\}_{k}$.

2) Since $\hat{\varphi}^{*}(\omega)$ and $G_{\varphi}(\omega)$ are $\operatorname{Lip}^{\varepsilon}$-functions, we have

$$
\left\|\hat{\varphi}^{*}(\omega) / G_{\varphi}(\omega)\right\|_{0}=\inf \left|\hat{\varphi}^{*}(\omega) / G_{\varphi}(\omega)\right|>0
$$

and

$$
\left\|\hat{\varphi}^{*}(\omega) G_{\varphi}(\omega)\right\|_{0}=\inf \left|\hat{\varphi}^{*}(\omega) / G_{\varphi}(\omega)\right|>0 .
$$

Therefore, (60) is equivalent to

$$
\delta_{\varphi}<\left(\frac{\inf \left|\hat{\varphi}^{*}(\omega) / G_{\varphi}(\omega)\right| \inf \left|\hat{\varphi}^{*}(\omega) G_{\varphi}(\omega)\right|}{\left\|q_{\varphi}(s, 0)\right\|_{L_{0}^{\lambda}[a, b]}}\right)^{1 / \lambda} .
$$

3) In the orthogonal case, $G_{\varphi}(\omega)$ is a constant. Then, (60) becomes

$$
\delta_{\varphi}<\left(\frac{\inf \left|\hat{\varphi}^{*}(\omega)\right|^{2}}{\left\|q_{\varphi}(t, 0)\right\|_{L_{0}^{\lambda}[a, b]}}\right)^{1 / \lambda} .
$$

Furthermore, if $\varphi(t)$ is also a cardinal scaling function, then $q_{\varphi}(t, 0)=\varphi(t)$ and $\hat{\varphi}^{*}(\omega)=1 \quad$ a.e. $\omega$. Therefore, (60) is the same as (19), i.e.,

$$
\delta_{\varphi}<\|\varphi\|_{L_{0}^{\lambda}[a, b]}^{-1 / \lambda} .
$$




\section{A Modified Version for General WaVElet Subspaces}

If $\hat{\varphi}^{*}(\omega) \neq 0$, we can apply the above algorithms to deal with irregularly sampled signals. Unfortunately, some scaling functions, even some important scaling functions, do not show the property. For example, consider the B-spline of order 2 scaling function

$$
\begin{aligned}
N_{2}(t)= & \frac{x^{2}}{2} \chi_{[0,1)}(t)+\frac{6 t-2 t^{2}-3}{2} \chi_{[1,2)}(t) \\
& +\frac{(3-t)^{2}}{2} \chi_{[2,3)}(t),
\end{aligned}
$$

where $\chi_{[j, j+1)}(t)$ is the characteristic function of the interval $[j, j+1)$ for $j=0,1,2$. Then $\hat{N}_{2}^{*}(\omega)=7 e^{i \omega}\left(e^{i \omega}+1\right) / 2=0$ when $\omega=\pi$. So we should find a proper way to solve it. This is the main purpose of this section.

Suppose the scaling function $\varphi(t)$ satisfies $|\varphi(t)| \leq$ $O\left(1 /|t|^{1+\varepsilon}\right)$ for some $\varepsilon>0$. Then, for $\sigma \in[0,1)$, we can define the Zak-transform of $\varphi$ (see [8], [9], and [17]) as

$$
Z_{\varphi}(\sigma, \omega)=\sum_{n} \varphi(\sigma+n) e^{i n \omega}
$$

For the above B-spline of order 2 scaling function $N_{2}(t)$, we find $Z_{N_{2}}(1 / 2, \omega)=\left(1+6 e^{i \omega}+e^{2 i \omega}\right) / 8 \neq 0$. This implies that we can improve our above-mentioned algorithms by sampling at $\{\sigma+k\}$ instead of $\{k\}$ for some $\sigma \in[0,1)$ with $Z_{\varphi}(\sigma, \omega) \neq 0$. First, we modify the theorem and algorithm for sampling in biorthogonal wavelet subspaces, then we deduce the modified results for that in general wavelet subspaces. Since the procedure is similar to the former sections except that $Z_{\varphi}(\sigma, \omega)$ takes the place of $\hat{\varphi}^{*}(\omega)$, here we will not show it in detail. Now we only display the three results without proofs.

Theorem 7: Suppose the continuous scaling function $\varphi(t)$ of MRA $\left\{V_{m}\right\}_{m}$ satisfies

1) $|\varphi(t)| \leq O\left(1 /|t|^{1+\varepsilon}\right)$ for some $\varepsilon>0$,

2) $Z_{\varphi}(\sigma, \omega) \neq 0$ for some $\sigma \in[0,1)$.

Then, there exists a $\delta_{\sigma, \varphi} \in(0,1]$, such that for any $\left\{\delta_{k}\right\}_{k} \subset$ $\left[-\delta_{\sigma, \varphi}, \delta_{\sigma, \varphi}\right]$, there is a sequence $\left\{S_{\sigma, k}(s)\right\}_{k} \subset V_{0}$ such that

$$
f(s)=\sum_{k} f\left(k+\sigma+\delta_{k}\right) S_{\sigma, k}(s)
$$

holds for any $f \in V_{0}$.

Theorem 8: Suppose the continuous scaling function $\varphi(t)$ of MRA $\left\{V_{m}\right\}_{m}$ satisfies

1) $|\varphi(t)| \leq O\left(1 /|t|^{1+\varepsilon}\right)$ for some $\varepsilon>0$,

2) $Z_{\varphi}(\sigma, \omega) \neq 0$ for some $\sigma \in[0,1)$,

3) $\varphi(t) \in L_{\sigma}^{\lambda}[a, b]$.

Then for any $\left\{\delta_{k}\right\}_{k} \subset\left[-\delta_{\sigma, \varphi}, \delta_{\sigma, \varphi}\right] \cap[a, b]$, there exists a sequence $\left\{S_{\sigma, k}(t)\right\}_{k} \subset V_{0}$ such that (70) holds if

$$
\delta_{\sigma, \varphi}<\left(\frac{\left\|Z_{\varphi}(\sigma, \omega) G_{\varphi}(\omega)\right\|_{0}\left\|Z_{\varphi}(\sigma, \omega) / G_{\varphi}(\omega)\right\|_{0}}{\left\|q_{\varphi}(s, \sigma)\right\|_{L_{\sigma}^{\lambda}[a, b]}}\right)^{1 / \lambda} .
$$

Corollary 4: In Theorem 8 , if 3 ) is replaced by "For a sequence $\left\{\delta_{k}\right\}_{k}$ there is a constant $C_{\varphi}>0$ such that for any mapping $\tau: Z \rightarrow Z$ ( $\mathrm{Z}$ is the integer set)

$$
\sum_{k} \mid \varphi\left(k+\sigma+\delta_{\tau(k)}-\left.\varphi(k+\sigma)\left|\leq C_{\varphi} \sup \right| \delta_{k}\right|^{\lambda}\right.
$$

for some $\lambda>0$,"

then there is a sequence $\left\{S_{\sigma, k}(s)\right\}_{k} \subset V_{0}$ such that (70) holds if

$$
\sup _{k}\left|\delta_{k}\right|<\left(C_{\varphi}^{-1}\left\|Z_{\varphi}(\sigma, \omega) G_{\varphi}(\omega)\right\|_{0}\left\|\frac{Z_{\varphi}(\sigma, \omega)}{G_{\varphi}(\omega)}\right\|_{0}\right)^{1 / \lambda}
$$

\section{Remark 4:}

1) The $\left\{S_{\sigma, k}(s)\right\}_{k}$ in Theorems 7 and 8 , and Corollary 9 are biorthogonal to $\left\{q_{\tilde{q}_{\sigma, 0}, q_{\varphi}(\cdot, \sigma)}\left(s, k+\sigma+\delta_{k}\right)\right\}_{k}$.

2) Since $Z_{\varphi}(0, \omega)=\hat{\varphi}^{*}(\omega)$, (71) is the same as (60) when $\sigma=0$.

3) We can also show that $Z_{\varphi}(\sigma, \omega) \in \operatorname{Lip}^{\varepsilon}$. Therefore, (71) is equivalent to

$$
\delta_{\sigma, \varphi}<\left(\frac{\inf \left|Z_{\varphi}(\sigma, \omega) G_{\varphi}(\omega)\right| \inf \left|Z_{\varphi}(\sigma, \omega) / G_{\varphi}(\omega)\right|}{\left\|q_{\varphi}(s, \sigma)\right\|_{L_{\sigma}^{\lambda}[a, b]}}\right)^{1 / \lambda} .
$$

\section{CONClusion AND EXAMPLES}

1) Suppose $\left\{V_{m}\right\}_{m}$ is a Multi-Resolution Decomposition of $L^{2}(R)$ with the scaling function $\varphi(t)$ satisfying

A. $|\varphi(t)| \leq O\left(1 /|t|^{1+\varepsilon}\right)$ for some $\varepsilon>0$.

B. $Z_{\varphi}(\sigma, \omega) \neq 0$ for some $\sigma \in[0,1)$.

We can assert that there is a $\delta_{\sigma, \varphi} \in(0,1]$ such that for any irregularly sampled points $\left\{k+\delta_{k}\right\}_{k}$ with $\sup _{k}\left|\delta_{k}\right|<\delta_{\sigma, \varphi}$, the original signal $f \in V_{0}$ can be reconstructed by

$$
f(s)=\sum_{k} f\left(k+\sigma+\delta_{k}\right) S_{\sigma, k}(s) .
$$

2) The $S_{\sigma, k}(s)$ in (73) can be computed as the biorthogonal

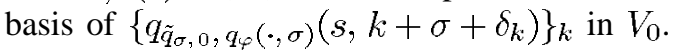

3) Besides the constraints $\mathbf{A}$ and $\mathbf{B}$, if the scaling function also satisfies

C. $\varphi(t) \in L_{\sigma}^{\lambda}[a, b],(\lambda>0,0 \in[a, b] \subset[-1,1])$ then we can estimate the deviation bound by

$$
\delta_{\sigma, \varphi}<\left(\frac{\inf \left|Z_{\varphi}(\sigma, \omega) G_{\varphi}(\omega)\right| \frac{\inf \left|Z_{\varphi}(\sigma, \omega)\right|}{G_{\varphi}(\omega)}}{\left\|q_{\varphi}(s, \sigma)\right\|_{L_{\sigma}^{\lambda}[a, b]}}\right)^{1 / \lambda} .
$$

4) For the irregularly sampled signals $\left\{f\left(k+\delta_{k}\right)\right\}_{k}$ we can also verify Corollary 5 to recover the original signal $f(t)$.

5) In fact, we have not used the dilation equation. Therefore, all the theorems are correct only with the hypothesis that $\{\varphi(t-n)\}_{n}$ is a Riesz basis of $V_{0}$ instead of that 
$\varphi(t)$ is a scaling function, i.e., only with the hypothesis of $0<\left\|G_{\varphi}(\omega)\right\|_{0} \leq\left\|G_{\varphi}(\omega)\right\|_{\infty}<\infty$.

6) If the sampling step is not at 1 or say $T=2^{-m}$, we can regard $V_{m}$ as $V_{0}$. All the theorems and algorithms can be modified to $V_{m}$ easily by using the Hilbert reproducing kernel

$$
q_{\varphi, \tilde{\varphi}}^{(m)}(s, t)=2^{m} \sum_{n} \varphi\left(2^{m} s-n\right) \tilde{\varphi}\left(2^{m} t-n\right) .
$$

Now we apply the algorithm to calculate some examples.

Example 1 (See [3]): Daubechies scaling function $\varphi_{N}(t)$ $(N=1,2,3, \cdots)$ is defined as

$$
\hat{\varphi}_{N}(\omega)=\prod_{1}^{\infty} H\left(2^{-n} \omega\right)
$$

where

$$
H(\omega)=\left(\left(1+e^{-i \omega}\right) / 2\right)^{N} M_{N}(\omega)
$$

and

$$
M_{N}(\omega)=\sum_{0}^{N-1} C_{N-1+n}^{n}\left(\sin ^{2} \omega / 2\right)^{n}
$$

It has been shown that $\varphi_{N}(t)$ is orthonormal, $\sup \varphi_{N} \subset$ $[0,2 N-1]$ and

$$
\varphi_{N}(t) \in \operatorname{Lip}^{(\min \{\mu N, 1\})}, \quad \mu=0.18 \text {. }
$$

Therefore, $\varphi_{N}(t) \in L_{\sigma}^{(\min \{\mu N, 1\})}[-1,1]$ for any $\sigma \in[0,1)$ due to Proposition 1 . If $Z_{\varphi_{N}}(\sigma, \omega) \neq 0$ for some $\sigma \in[0,1)$, then

$$
\delta_{\sigma, \varphi_{N}}<\left(\frac{\inf _{\omega}\left|Z_{\varphi_{N}}(\sigma, \omega)\right|}{2 N\left\|\varphi_{N}\right\|_{\operatorname{Lip}}(\min \{\mu N, 1\})}\right)^{1 /(\min \{\mu N, 1\})} .
$$

Example 2 (See [15]): Meyer scaling function is defined as

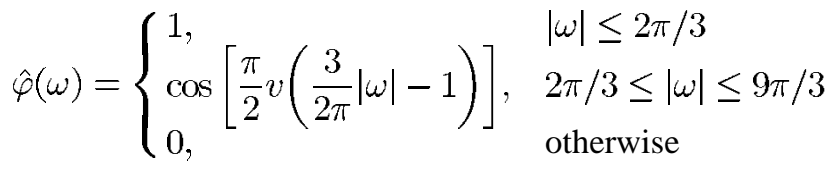

where $v(\omega) \in C^{\infty}, v(\omega)=1$ when $\omega \geq 1, v(\omega)=0$ when $\omega \leq 0$, and $v(\omega)+v(1-\omega)=1$. It is shown that $\varphi(t)$ is orthonormal and $r$-regular. Therefore,

$$
\sum_{n} \sup _{[n, n+1]}\left|\varphi^{\prime}(t)\right|
$$

converges. Hence $\varphi(t) \in L_{\sigma}^{1}[-1,1]$ for any $\sigma \in[0,1)$ due to Proposition 1. Since

$$
\begin{gathered}
\inf \left|\hat{\varphi}^{*}(\omega)\right|=\min \left\{1, \inf _{-4 \pi / 3 \leq \omega \leq-2 \pi / 3} \mid \cos \left(\frac{\pi}{2} v\left(\frac{3 \omega}{2 \pi}+2\right)\right)\right. \\
\left.+\cos \left(\frac{\pi}{2} v\left(-\frac{3 \omega}{2 \pi}-1\right)\right) \mid\right\}=1
\end{gathered}
$$

we obtain

$$
\delta_{\varphi}<\left(\sum_{n} \sup _{[n, n+1]}\left|\varphi^{\prime}(t)\right|\right)^{-1} .
$$

The following example indicates that $\delta_{\varphi}$ can be bigger than $1 / 4$ for the sampling in the B-spline of order 1 wavelet subspace.

Example 3 (See [1]): The B-spline of order 1 scaling function

$$
N_{1}(t)=t \chi_{[0,1)}+(2-t) \chi_{[1,2)} .
$$

Obviously, $N_{1}(t)$ satisfies constraint $\mathbf{A}$ and $\mathbf{C}$ (with $\sigma=0$, $\lambda=1$ ). Since $\hat{N}_{1}^{*}(\omega)=1$

$$
G_{N_{1}}(\omega)=\left(1 / 3+2 / 3 \cos ^{2}(\omega / 2)\right)^{1 / 2}
$$

and

$$
\sum_{k} N_{1}(t+k) N_{1}(k)=N_{1}(t) .
$$

Therefore, (74) becomes

$$
\frac{\left\|\left(\frac{1}{3}+\frac{2}{3} \cos ^{2}\left(\frac{\omega}{2}\right)\right)^{1 / 2}\right\|\left\|_{0}\right\| \frac{1}{\left(\frac{1}{3}+\frac{2}{3} \cos ^{2}\left(\frac{\omega}{2}\right)\right)^{1 / 2}} \|_{0}}{\left\|N_{1}\right\|_{L_{0}^{1}[a, b]}}
$$

Since

$\left\|N_{1}\right\|_{L_{0}^{1}[-1,1]}=3,\left\|N_{1}\right\|_{L_{0}^{1}[-1,0]}=2$, and $\left\|N_{1}\right\|_{L_{0}^{1}[0,1]}=2$ we derive $\delta_{\varphi}<1 / 3 \sqrt{3}$. When $\delta_{k} \leq 0$ for all $k$ or $\delta_{k} \geq 0$ for all $k$, we have $\delta_{\varphi}<1 / 2 \sqrt{3}$. Obviously, $1 / 2 \sqrt{3}>1 / 4$.

\section{ACKNOWLEDGMENT}

The authors wish to thank the referees as well as Prof. G. G. Walter for their good suggestion. They also wish to thank Prof. R. Long, Prof. T. Hashimoto, and Dr. Y. Zhou for their partial contributions.

\section{REFERENCES}

[1] C. K. Chui, "An introduction to wavelets," in Wavelet Analysis and Its Applications, vol. 1. New York: Academic, 1992.

[2] W. Chen, S. Itoh, and J. Shiki, "Sampling theorems by wavelets for irregularly sampled signals," IEICE Trans., vol. J79-A, no. 12, pp. 1991-1997, 1996.

[3] I. Daubechies, "Orthonormal bases of compactly supported wavelets," Commun. Pure Appl. Math., vol. 91, no.7, pp. 909-996, 1988.

[4] - "The wavelets transform, time frequency localization, and signal analysis," IEEE Trans. Inform. Theory, vol. 36, pp. 961-1005, Sept. 1990.

[5] I. Daubechies, A. Grossmann, and Y. Meyer, "Painless nonorthogonal expansions," J. Math. Phys., vol. 27, pp. 1271-1283, 1986.

[6] R. E. Edwards, "Fourier series," in Graduate Texts in Math. 69 and 85. Berlin, Germany: Springer-Verlag, 1979 and 1981.

[7] H. Feichtinger and K. Grochenig, "Theory and practice of irregular sampling," in Wavelets: Mathematics and Applications, J. Benedetto and M. Frazier, Eds. Boca Raton, FL: CRC, 1993.

[8] C. E. Heil and D. F. Walnut, "Continuous and discrete wavelet transforms," SIAM Rev., vol. 31, pp. 628-666, 1989. 
[9] A. J. E. M. Janssen, "The Zak transform and sampling theorem for wavelet subspaces," IEEE Trans. Signal Processing, vol. 41, pp. 3360-3369, Dec. 1993

[10] Y. Liu, "Irregular sampling for spline wavelet subspaces," IEEE Trans. Inform. Theory, vol. 42, pp. 623-627, Mar. 1996.

[11] Y. Liu and G. G. Walter, "Irregular sampling in wavelet subspaces," $J$. Fourier Anal. Appl., vol. 2, no. 2, pp. 181-189, 1995.

[12] R. Long and D. Chen, "Biorthogonal wavelet bases on $R^{d}$," Appl. Comp. Harmonic Anal., vol. 2, pp. 230-292, 1995.

[13] R. Long and W. Chen, "Wavelet basis packet and wavelet frame packet," J. Fourier Anal. Appl., vol. 3, no. 3, pp. 229-256, 1997.
[14] R. Long, W. Chen, and S. Yuan, "Wavelet generated by vector multiresolution analysis," Appl. Comput. Harm. Anal., vol. 9, pp. 317-350, 1997.

[15] Y. Meyer, "Wavelets and operators," in Cambridge Studies in Advanced Math. 37. London, U.K.: Cambridge Univ. Press, 1992.

[16] G. G. Walter, "A sampling theorem for wavelet subspaces," IEEE Trans. Inform. Theory, vol. 38, pp. 881-889, Mar. 1992.

[17] Wavelets and Orthogonal System with Applications. Boca Raton, FL: CRC, 1994

[18] R. M. Young, An Introduction to Non-Harmonic Fourier Series. New York: Academic, 1980 